\title{
Patrones y factores subyacentes de la estructura y dinámica de bosques Mediterráneos: implicaciones teóricas y aplicadas
}

\author{
P. Ruiz-Benito ${ }^{1,2,3, *}$ \\ (1) Grupo de Ecología y Restauración Forestal, Departamento de Ciencias de la Vida, Unidad Docente de Ecología, Edificio de Ciencias, Universidad de \\ Alcalá, Campus Universitario, 28805, Alcalá de Henares (Madrid), España. \\ (2) CIFOR-INIA. Ctra. de la Coruña, Km. 7,5. 28040. Madrid, Spain. \\ (3) Biological and Environmental Sciences, School of Natural Sciences. University of Stirling, FK9 4LA, Stirling, United Kingdom.
}

* Autor de correpondencia: P. Ruiz-Benito [paloma.ruizbenito@stir.ac.uk]

> Recibido el 19 de noviembre de 2013, aceptado el 20 de noviembre de 2013.

Ruiz-Benito, P. 2013. Patrones y factores subyacentes de la estructura y dinámica de bosques Mediterráneos: implicaciones teóricas y aplicadas. Ecosistemas 22(3):128-130. Doi.: 10.7818/ECOS.2013.22-3.18

Los bosques cubren más del $30 \%$ de la biosfera, albergan cerca de dos tercios de la diversidad biológica y proporcionan múltiples servicios ecosistémicos (Millennium Ecosystem Assessment 2005; FAO 2012). Sin embargo, el cambio global está causando importantes modificaciones tanto en la extensión de los ecosistemas naturales como en la cantidad y calidad de los servicios ecosistémicos proporcionados (Fig. 1). Ya se han observado alteraciones en las tasas demográficas básicas de las especies arbóreas (i.e. regeneración, crecimiento, mortalidad) que podrían traducirse en alteraciones en su distribución, así como en variaciones de la cantidad y calidad de los servicios ecosistémicos proporcionados por los bosques (Fig. 2). Por tanto, cuanto mayor sea nuestro conocimiento de la importancia relativa de los distintos factores que dirigen la demografía de las especies arbóreas, mayor será nuestra capacidad de inferir las implicaciones del cambio global para los ecosistemas forestales. El objetivo general de la presente tesis doctoral es analizar los factores bióticos y abióticos determinantes de procesos demográficos (i.e. regeneración, mortalidad y crecimiento; primera parte) y servicios ecosistémicos clave (i.e. productividad y almacenamiento de carbono; segunda parte) en los bosques Ibéricos, con el fin último de inferir efectos potenciales del cambio global sobre su estructura y dinámica (Tabla 1).

En la primera parte de la tesis estudié la variación de procesos demográficos (i.e. regeneración, crecimiento y mortalidad) a lo largo de gradientes bióticos y abióticos amplios, y sus efectos sobre los patrones de distribución de especies arbóreas en un contexto de cambio global usando información del segundo y tercer Inventario Forestal Español (IFN). Primero, analicé patrones de regeneración y diversidad en pinares de repoblación de la península ibérica en comparación con los patrones observados en pinares naturales, a lo largo de gradientes bióticos y abióticos (Ruiz-Benito et al. 2012). Los pinares plantados presentaron menor regeneración y diversidad que los pinares naturales, presumiblemente debido a las diferentes características estructurales encontradas. Las máximas diferencias en regeneración y diversidad entre pinares plantados y naturales ocurrieron a niveles bajos-medios de densi- dad, de frecuencia de incendios, de precipitación y de distancia a bosques de Quercus. En segundo lugar, examiné la importancia relativa del tamaño del árbol, la competencia y del clima en la mortalidad arbórea para las 11 especies arbóreas más abundantes de la península ibérica (Ruiz-Benito et al. 2013). Las tasas de mortalidad se vieron positivamente afectadas por la competencia asimétrica, sobre todo en coníferas.

Consistentemente a lo largo de este primer bloque de la tesis doctoral encontré que la estructura del rodal fue un factor clave, y relativamente más importante que el clima, determinante del éxito de los diferentes procesos demográficos (Fig. 3). Además, las distintas tasas demográficas variaron en su respuesta a las características estructurales del dosel. Por ejemplo, a lo largo de un gradiente de densidad arbórea, los máximos de regeneración tuvieron lugar a niveles de densidad, y por tanto de competencia, intermedios (Ruiz-Benito et al. 2012), los de crecimiento a niveles de competencia bajos (Gómez-Aparicio et al. 2011), y los de mortalidad a niveles de competencia altos (Ruiz-Benito et al. 2013). El hecho de que la mortalidad y el crecimiento presentaran tendencias opuestas, sugiere que bajo competencia intensa las especies podrían estar funcionando en su límite fisiológico, conllevando bajos niveles de crecimiento y elevadas tasas de mortalidad (Franklin et al. 1987; Valladares y Pearcy 2002).

En cuanto al clima, la importancia relativa de la temperatura como determinante de las tasas demográficas fue mayor que la de la precipitación, generalmente observando mayores tasas de mortalidad a elevadas temperaturas (Ruiz-Benito et al. 2013), lo que probablemente se debe a un mayor estrés hídrico inducido por altas temperaturas (e.g. Carnicer et al. 2011). Además, uno de los resultados más novedosos de la presente tesis doctoral es la existencia de una importante interacción entre los efectos del clima y de la estructura del rodal sobre la mortalidad (Ruiz-Benito et al. 2013). Un aumento de la aridez bajo cambio climático podría causar elevadas mortalidades (Ruiz-Benito et al. 2013) y crecimientos reducidos en los bosques de alta densidad, con serias consecuencias para la estructura de las comunidades (Gómez-Aparicio et al. 


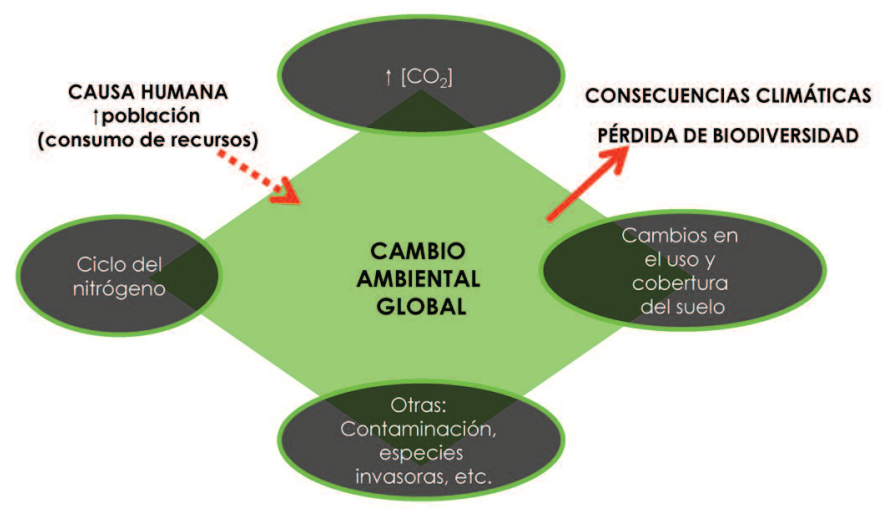

Figura 1. Marco del cambio ambiental global, en el que se determinan los principales componentes - aumento de la concentración de dióxido de carbono atmosférico, cambios en el ciclo del nitrógeno, cambios en el uso y cobertura del suelo, y otros componentes-. Las principal causa del cambio global es el aumento de la población humana, que tiene una serie de consecuencias climáticas y efectos negativos sobre la diversidad (ver Vitousek 1994).

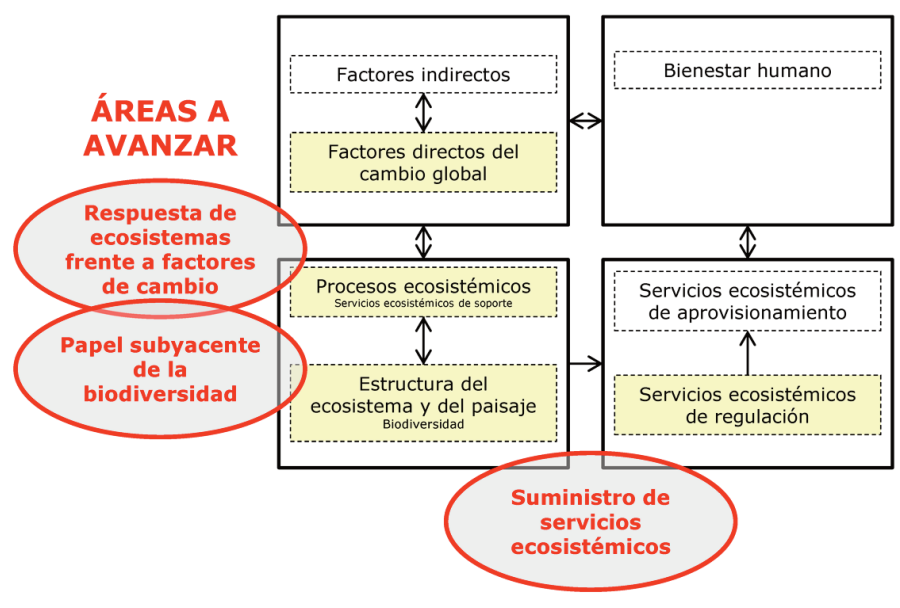

Figura 2. Circuito de retroalimentación general considerando cómo factores directos (e.g. cambio climático o cambios en el uso del suelo) e indirectos (e.g. cambios en el sistema socio-económico) modifican el funcionamiento del ecosistema (i.e. los procesos ecosistémicos y la biodiversidad) y estos a su vez el aprovisionamiento y regulación de servicios ecosistémicos y el bienestar humano. Modificado de Carpenter et al. (2009) y Evaluación de Ecosistemas del Milenio (Millennium Ecosystem Assessment 2005). Los recuadros en amarillo son las áreas de conocimiento en las que se ha avanzado en la presente tesis doctoral, las cuales se relacionan con las "áreas a avanzar" para mejorar el conocimiento teórico y aplicado del funcionamiento y respuestas de los bosques a factores de cambio y biodiversidad (UNEP 2008). "Factor" se define como cualquier factor natural o inducido por el ser humano que directa o indirectamente produzca un cambio en un ecosistema.

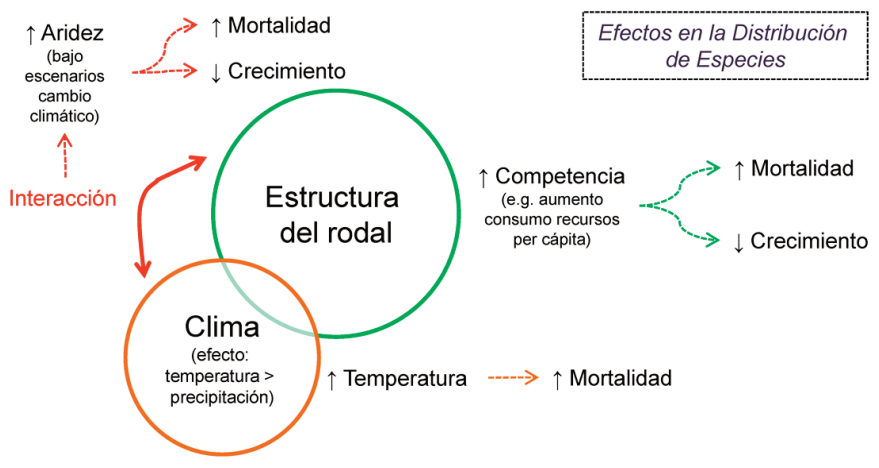

Figura 3. Esquema conceptual de la importancia relativa de la estructura del rodal y el clima (donde la importancia relativa se muestra con el tamaño relativo del círculo) en el crecimiento y la mortalidad. Se muestran los efectos observados en el crecimiento y la mortalidad, tanto directos como por la interacción entre factores (Gómez-Aparicio et al. 2011; Ruiz-Benito et al. 2013), lo que podría modificar estas tasas bajo cambio climático y causar cambios en la distribución de especies (Benito-Garzón et al. 2013).
2011). En un último trabajo de esta primera parte de la tesis los resultados modelos de nicho parametrizados con crecimiento y mortalidad de especies arbóreas presentes en la península ibérica, sugieren que bajo escenarios de cambio climático tanto el crecimiento como la mortalidad pueden verse incrementados, causando modificaciones en los rangos de distribución de las especies (Benito-Garzón et al. 2013).

En conjunto, mis resultados sugieren que bajo escenarios de cambio climático donde se produzca un aumento generalizado de la aridez los bosques de alta densidad serían particularmente susceptibles a sufrir colapsos demográficos, independientemente de su composición, pues todas las especies arbóreas se vieron profundamente afectas por la competencia bien directamente (como las coníferas) o indirectamente a través de su interacción con el clima (en el caso de las planifolias). Sin embargo, en bosques de densidad intermedia las diferencias inter-específicas en la respuesta al clima serían más patentes. Así, las especies con el límite sur de su distribución en la península ibérica (e.g. especies de montaña) serían más sensibles a reducciones de regeneración, supresión del crecimiento e incrementos de mortalidad bajo escenarios de cambio climático que las especies Mediterráneas, mejor adaptadas a condiciones climáticas de aridez.

En la segunda parte de la tesis estudié el efecto de la diversidad, la estructura del rodal, el clima y el cambio climático reciente en el suministro de servicios ecosistémicos clave por los sistemas forestales. Primero, analicé el papel de la diversidad y los mecanismos subyacentes en el almacenamiento de carbono y productividad en una amplia variedad de bosques Ibéricos (desde bosques Mediterráneos hasta bosques templados Atlánticos; Ruiz-Benito et al., en prensa). La diversidad funcional afectó directamente a ambos servicios ecosistémicos, mediante mecanismos de complementariedad y selección. Los mecanismos de complementariedad tuvieron efectos positivos en todos los tipos de bosque, mientras que los mecanismos de selección fueron particularmente importantes en pinares Mediterráneos y bosques caducifolios. Finalmente, estudié las tendencias de cambios en biomasa en bosques Europeos pertenecientes a los biomas Mediterráneo, templado y boreal; y examiné el efecto de la estructura de la parcela, el clima y el cambio climático reciente (i.e. cambios en temperatura o precipitación entre dos inventarios consecutivos; Ruiz-Benito et al., datos sin publicar). Observé un incremento en biomasa durante las últimas décadas del s. XX en bosques Europeos, particularmente positivo en bosques templados. Los bosques relativamente jóvenes (con tamaños y área basal bajos-medios) presentaron los mayores incrementos en biomasa, pero también fueron los más sensibles a condiciones climáticas adversas. El cambio climático reciente (principalmente aumentos en temperatura) tuvo un efecto positivo en los incrementos en biomasa, pero que se volvió nulo o negativo en zonas áridas limitadas por la disponibilidad de agua (e.g. gran parte bosques Mediterráneos). Por tanto, mis resultados sugieren que los bosques con mayor potencial para actuar como sumidero de carbono serían los bosques relativamente jóvenes no limitados por agua ni energía, si bien este efecto sumidero podría verse constreñido por los efectos del cambio climático reciente.

\section{Agradecimientos}

La presente tesis doctoral ha sido financiada por una beca F.P.U. (AP2008-01325), por los Proyectos FUNDIV (ENV.2010.2.1.4-1), Carbo-Extreme (FP7-ENV-2008-1-2267), INTERBOS3-CGL200804503-C03-03, SUM2008-00004-C03-01 y REMEDINAL-2. Especial agradecimiento al MAGRAMA por el acceso a los datos del Inventario Forestal Nacional, a los lugares y personas con las que se ha desarrollado la presente tesis doctoral: Departamento de Ciencias de la Vida (Universidad de Alcalá), Centro de Investigación Forestal (CIFOR-INIA), Instituto de Recursos Naturales y Agrobiología (IRNAS-CSIC), Plant Sciences Department (Universidad de Cambridge), Department des Sciences Biologuiques (Université du Québec à Montreal). 
Tabla 1. Resumen de objetivos, material y métodos y resultados en forma de publicación de los capítulos de la presente tesis doctoral.

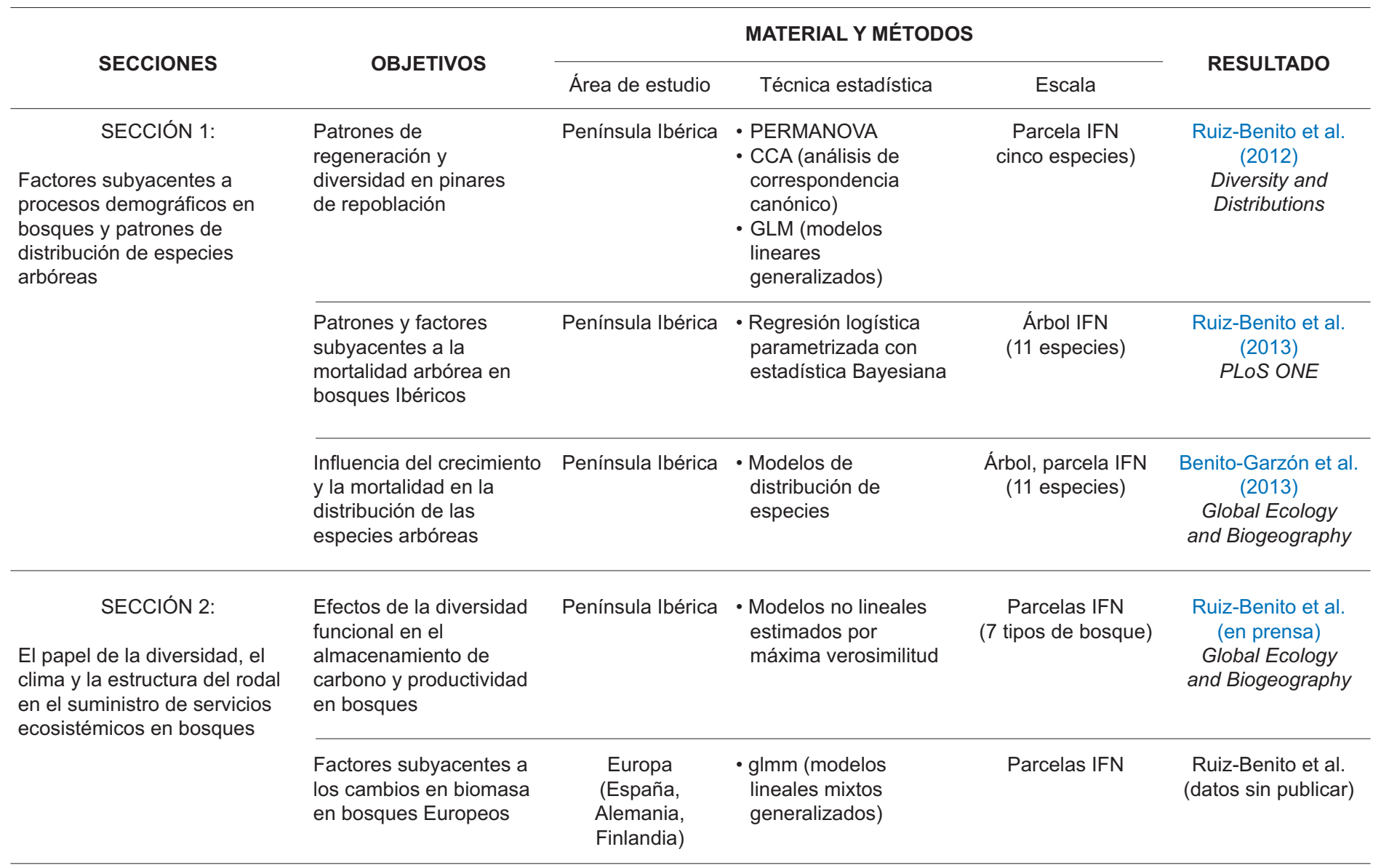

IFN: Inventario Forestal Nacional.

\section{Referencias}

Carnicer, J., Coll, M., Ninyerola, M., Pons, X., Sánchez, G., Peñuelas, J. 2011. Widespread crown condition decline, food web disruption, and amplified tree mortality with increased climate change-type drought. Proceedings of the National Academy of Sciences 108: 14741478.

Carpenter, S.R., Mooney, H.A., Agard, J., Capistrano, D., DeFries, R.S., Díaz, S., Dietz, T., Duraiappah, A.K., Oteng-Yeboah, A., Pereira, H.M., Perrings, C., Reid, W.V., Sarukhan, J., Scholes, R.J., Whyte, A. 2009. Science for managing ecosystem services: Beyond the Millennium Ecosystem Assessment. Proceedings of the National Academy of Sciences 106: 1305-1312.

FAO 2012. State of the world's forests. Food and Agriculture Organization of the United Nations. Roma, Italia.

Franklin, J.F., Shugart, H.H., Harmon, M.E. 1987. Tree death as an ecological process. Bioscience 37: 550-556.

Gómez-Aparicio, L., García-Valdés, R., Ruiz-Benito, P., Zavala, M.A. 2011. Disentangling the relative importance of climate, size and competition on tree growth in Iberian forests: implications for management under global change. Global Change Biology 17: 2400-2414.

Millennium Ecosystem Assessment 2005. Ecosystem and human wellbeing: biodiversity synthesis. World Resources Institute. Washington D.C., Estados Unidos.

UNEP 2008. The Millennium Ecosystem Assessment (MA) follow-up: A global strategy for turning knowledge into action. UNEP/CBD/COP/ 9/INF/26. Bonn, Alemania.

Valladares, F., Pearcy, R.W. 2002. Drought can be more critical in the shade than in the sun: a field study of carbon gain and photo-inhibition in a Californian shrub during a dry El Niño year. Plant, Cell and Environment 25: 749-759.

Vitousek, P.M. 1994. Beyond global warming: Ecology and global change. Ecology 75: 1861-1876.

\section{PALOMA RUIZ-BENITO}

Patrones y factores subyacentes de la estructura y dinámica de bosques Mediterráneos: implicaciones teóricas y aplicadas

Departamento de Ciencias de la Vida, Universidad de Alcalá. Tesis Doctoral 16 Mayo 2013

Directores: Miguel A. Zavala, Lorena Gómez-Aparicio

\section{Publicaciones resultantes de la tesis}

Benito-Garzón, M., Ruiz-Benito, P., Zavala, M.A. 2013. Inter-specific differences in tree growth and mortality responses to climate determine potential species distribution limits in Iberian forests. Global Ecology and Biogeography 22: 1141-1151.

Ruiz-Benito, P., Gómez-Aparicio, L., Paquette, A., Messier, C., Kattge, J., Zavala, M.A. (en prensa) Diversity increases carbon storage and tree productivity in Iberian forests. Global Ecology and Biogeography. doi: 10.1111/geb.12126.

Ruiz-Benito, P., Gómez-Aparicio L., Zavala M.A. 2012. Large scale assessment of regeneration and diversity in Mediterranean planted pine forests along ecological gradients. Diversity and Distributions 18: 1092-1106.

Ruiz-Benito, P., Lines, E.R., Gómez-Aparicio, L., Zavala M.A, Coomes DA. 2013. Patterns and drivers of tree mortality in Iberian forests: climatic effects are modified by competition. PLoS ONE 8: e56843. 